\title{
Transseptal Puncture for Atrial Fibrillation Ablation Under Electroanatomic Guidance: Is it Feasible?
}

\author{
Khaled Sabeh ${ }^{1}$ and Marwan Refaat ${ }^{2}$ \\ ${ }^{1}$ Massachusetts General Hospital \\ ${ }^{2}$ American University of Beirut Medical Center
}

July 20, 2020

The field of electrophysiology continues to move further towards low fluoroscopy procedures. The deleterious effects of radiation exposure and of the radiation protection clothing themselves are the primary drivers of this approach. Radiation exposure is known to increase the risk of cancer and cataracts for all operators, and namely those who are subjected to accumulating doses of radiation over time. (1). Proper radiation protective clothing can significantly decrease these risks however this strategy has serious weaknesses. For instance, the protective clothing does not cover the whole body, leaving the face and the skull exposed. Roguin et al (2) showed that the risk of radiation exposure to the unprotected areas of the body is real and has serious consequences. In a cohort of 31 interventional cardiologists who developed brain cancer, the investigators showed that $22(85 \%)$ of them had left sided tumors, and $17(55 \%)$ of them had glioblastoma multiforme. This remarkable finding suggests that the dose left side of the brain, the side that gets more radiation exposure, is much more likely to develop a cancer that carries a poor prognosis and a median expected survival of 12 months. Furthermore, the radiation protective clothing itself can cause orthopedics injuries common among interventional cardiologists such those of the spine and the knees. Given the deleterious effects of radiation, low fluoroscopy approaches are welcomed by the electrophysiology community if they can show a safety profile similar to that with the use of fluoroscopy.

The transseptal puncture (TSP) is arguably the most critical step during which fluoroscopy is used. In this study Singh et al describe an approach for TSP under electoanatomic guidance. The authors then retrospectively compare the total procedure duration, fluoroscopy time, radiation exposures, and complications related to the TSP using this method with those of conventional fluoroscopy. This was a single center study that included 145 consecutive patients, with no previous history of cardiac surgery, who underwent de novo and redo AF ablations between June 2018 and April 2019. These patients were then compared to cases performed by the same operators before June 2018. The procedure was done under conscious sedation. A dense electroanatomic map of the right atrium was acquired using CARTO 3 Fast Anatomical Mapping and Confidence Software, with emphasis on the atrial septum, His Bundle, coronary sinus ostium, and superior vena cava. The authors observed that the fossa ovalis was an area of low voltage potential $(0.37 \pm 0.19 \mathrm{mV}$ vs $1.73 \pm 0.74 \mathrm{mV})$ and low impedance $(125 \pm 11 \Omega$ vs $138 \pm 15 \Omega)$, and electrically distinct from the rest of the atrial septum. The authors were able to localize the fossa ovalis using a combination of anatomical landmarks and the use of a voltage threshold of $0.75 \mathrm{mV}$. The transseptal needle was then advanced through this desired location. The authors reported no significant complications related to the TSP.

The authors argue that the safety profile is like the TSP under fluoroscopy, however this is a single center study. In fact the most senior operator performed three- quarters of all the procedures. Given the high risk of such an approach, the main question for the wide adoption of such a technique will again be safety in the hands of less experienced operators. A major factor that can increase the safety profile as well as the 
preciseness of the TSP is the routine use of ICE. ICE can confirm the precise positioning of the needle even in cases with unusual atrial septal anatomies (floppy, bulging, hypertrophic septum or in the presence of devices such as CardioSEAL or other atrial septal defect occlusion devices). Furthermore, ICE can confirm the location of the needle in the LA with microbubble injections after the TSP; it can confirm the location of the wire thus making it safer to advance the sheath knowing that it will not end up in the LAA or causing a perforation. As such ICE is arguably more important in the low fluoroscopy approach than in a one with fluoroscopy.

Low fluoroscopy approach to TSP is a welcomed change in the field of electrophysiology given the significant adverse outcomes of radiation and radiation protective clothing to providers. The main concern in such a change is the safety and precise localization of the TSP. New technologies are allowing the development of new approaches such as the one described by Troisi et al to achieve the goal of safe low fluoroscopy procedures.

\section{References:}

1. Klein LW, Miller DL, Balter S, et al. Occupational health hazards in the interventional laboratory: time for a safer environment. Radiology 2009; 250:538-544.

2. Roguin A, Goldstein J, Bar O, Goldstein JA. Brain and neck tumors among physicians performing interventional procedures. Am J Cardiol 2013;111(9):1368-72. 